\title{
Search for gluon saturation effects at the LHC with the ALICE experiment
}

\section{Cynthia Hadjidakis ${ }^{* \dagger}$}

Institut de Physique Nucléaire d'Orsay (IPNO)

CNRS: UMR8608 IN2P3 Université Paris Sud - Paris XI

15 Rue G. Clemenceau, 91406 Orsay, France

E-mail: Cynthia.Hadjidakisecern.ch

Gluon saturation effects are expected to occur at small parton momentum fraction Bjorken- $x$, affecting particle production in heavy ion collisions. In these proceedings, we focus on heavy flavour production in the forward rapidity region in $\mathrm{p}-\mathrm{p}$ and $\mathrm{p}-\mathrm{Pb}$ collisions as a tool to search for saturation effects. We will discuss some aspects of the detection performance of the ALICE Forward Muon Spectrometer.

XLVIII International Winter Meeting on Nuclear Physics, BORMIO2010

January 25-29, 2010

Bormio, Italy

\footnotetext{
*Speaker.

${ }^{\dagger}$ For the ALICE collaboration
} 


\section{Saturation and the Color Glass Condensate}

In this part, we summarize the saturation phenomenon in the nuclear matter and the theoretical framework which allows to describe it (for a recent review, see [1] and references therein). When probing small distances inside a hadron with a hard process, one resolves its partonic content. Extensive Deep Inelastic Scattering measurements were carried out at HERA to determine precisely parton distribution functions in the framework of the collinear factorization approach of perturbative QCD. In particular, the $Q^{2}$-evolution of these data allows an extraction of gluon distribution functions. At large energy (or at small Bjorken- $x$, the momentum fraction of the nucleon carried by the parton), the gluon density in the nucleon is seen to continuously rise. At a certain energy scale, the large gluon density in the transverse plane of the nucleon is expected to favour the gluon recombination process. This process would then limit a further growth of the gluon density. This scale, named the saturation scale $Q_{s}^{2}$, defines the boundary between a dilute and a saturation domain. In the dilute domain, partons interact independently with the probe and the linear DGLAP and BFKL evolution equations describe the $Q^{2}$ - and $x$-evolution of the nucleon structure, respectively. In the saturated domain, partons interact coherently with the probe and, due to recombination processes, the growth of the gluon density is governed by non-linear evolution equations such as JIMWLK or BK. In order to describe the hadronic matter in the saturation domain, the Color Glass Condensate (CGC) framework has been proposed. At sufficiently large $Q_{s}^{2}$, the theory is weakly coupled, although not perturbative due to the high gluon occupancy: it is a classical effective field theory.

\section{Saturation in heavy ion collisions}

When colliding two ultra-relativistic heavy ions, the 'hot' matter formed is expected to be deconfined and to form a quark gluon plasma. However both nuclei can be described as coherent and high density gluon systems and some of the observed effects in heavy ion collisions may come from gluon saturation effects in the incident nuclei. Understanding the initial state is then crucial in order to disentangle hot deconfined matter effects from cold nuclear matter ones. For that purpose, one can use experimentally nucleon-nucleus ( $\mathrm{p}-\mathrm{A}$ ) collisions to measure cold nuclear matter effects (note that cold nuclear effects include both initial and final state effects). At RHIC, d-Au collisions at $\sqrt{s}=200 \mathrm{MeV}$ provided an estimation of the cold nuclear matter effects through the measurement of the nuclear modification factor defined as: $R_{d A u}\left(y, p_{T}\right)=\frac{d N_{d A u} / d y d p_{T}}{\left\langle N_{c o l l}\right) d N_{p p} / d y d p_{T}}$ where $N_{d A u}\left(N_{p p}\right)$ is the yield of particles produced in d-Au (p-p) collisions and $\left\langle N_{\text {coll }}\right\rangle$ is the average number of binary collisions. This observable is supposed to be equal to unity if the d-Au collision is a superposition of independent nucleon-nucleon collisions. The BRAHMS experiment observed a large suppression for charged particles in d-Au compared to p-p collisions at forward rapidity and low $p_{T}$ [2]. This behaviour is described by the CGC model and these measurements represent a possible onset of the gluon saturation at the RHIC energies. However the saturation scale estimated at RHIC for Au is approximately $Q_{s}^{2}=2-4 \mathrm{GeV}^{2}$ for the pseudo-rapidity domain $|\eta|<3.2$ : the saturation domain is close to the non perturbative one. The LHC, with a maximum energy per nucleon in the centre of mass of $14,8.8$ and $5.5 \mathrm{TeV}$ for $\mathrm{p}-\mathrm{p}, \mathrm{p}-\mathrm{Pb}$ and $\mathrm{Pb}-\mathrm{Pb}$ collisions, respectively, will probe partons with very low $x$ values down to $10^{-6}$. The saturation scale will reach values up to $Q_{s}^{2}=5 \mathrm{GeV}^{2}$ for $\mathrm{p}$ and $Q_{s}^{2}=15 \mathrm{GeV}^{2}$ for $\mathrm{Pb}$. It will thus be possible to use perturbative probes 
to explore the saturation domain, providing definitive tests of the CGC picture. The LHC started in november 2009 with its first p-p collisions at $\sqrt{s}=900 \mathrm{GeV}$ and $\sqrt{s}=2.36 \mathrm{TeV}$. It will continue in 2010 with p-p collisions at $\sqrt{s}=7 \mathrm{TeV}$ and with its first $\mathrm{Pb}-\mathrm{Pb}$ collisions at $\sqrt{s_{N N}}=2.76 \mathrm{TeV}$. Collisions in $\mathrm{p}-\mathrm{Pb}$ are expected in the following years. ALICE is the LHC experiment dedicated to the study of the quark gluon plasma and to the heavy ion collisions. It consists of a central part lying in the L3 magnet and, in the forward region, of a spectrometer allowing to detect muons for the range $-4<\eta<-2.5$. The spectrometer consists of a passive frontal absorber in order to absorb hadrons from the interaction vertex, a large dipole magnet, a high granularity tracking system of ten detection planes and a passive muon filter wall followed by four planes of trigger chambers. A detailed description of the ALICE experiment can be found in [3]. In the following, we focus on heavy flavour production in the forward region as a tool to search for saturation effects.

\section{Forward heavy flavour pair production in the CGC framework}

Forward heavy flavour pair production has been calculated within the CGC framework [4]. At forward rapidity, the parton probed in the first projectile during the interaction has a large $x$ value. Both in $\mathrm{p}-\mathrm{p}$ and $\mathrm{p}-\mathrm{Pb}$ collisions, this projectile can then be considered as a dilute system. The corresponding $x$ value for the parton in the second projectile is much lower and lies in the saturation domain: this projectile is considered as a dense system. At LHC energies, gluon fusion processes dominate the heavy quark pair production which can be schematically written as:

$$
\frac{d N_{Q \bar{Q}}^{P P D}}{d y d^{2} \mathbf{p}_{\mathbf{T}}}=f_{g}^{(p)}\left(x_{1}\right) \otimes \phi\left(x_{2}\right) \otimes \frac{d N_{g g \rightarrow Q \bar{Q}}}{d y d^{2} \mathbf{p}_{\mathbf{T}}},
$$

where $f_{g}^{(p)}\left(x_{1}\right)$ is the usual gluon distribution in the proton, $\phi\left(x_{2}\right)$ is the gluon distribution describing the saturated nucleus, and $\frac{d N_{g g \rightarrow Q} \bar{Q}}{d y d^{2} \mathbf{p}_{\mathrm{T}}}$ is the differential heavy quark pair production from gluon fusion derived in a saturated system. For the second projectile, a constant saturation scale, related to the projectile density, is defined at $x=0.01$. A fixed value for the saturation scale implies that the thickness of the projectile is considered to be constant and collision centrality dependence is not taken into account. The results presented here correspond then to the most central collision. The saturation scale is defined from the measurement of the charged particle multiplicity in $\mathrm{Au}-\mathrm{Au}$ collisions at RHIC and is taken to be $2 \mathrm{GeV}^{2}$ for the $\mathrm{Pb}$ at $x=0.01$. The small $x$-evolution of the projectile density is estimated accordingly to the BK evolution equation.

\section{Open heavy flavour production and detection performance with the ALICE Muon Spectrometer}

From Eq. 3.1, open charm and beauty production has been derived both in p-p and p- $\mathrm{Pb}$ collisions. The nuclear modification ratio as a function of $p_{T}$ is shown in the left plot of Fig. 1 for the charm (in red) and beauty (in blue) production at forward rapidity. The CGC calculations (full lines) show a stronger suppression for the open heavy flavour production in $\mathrm{p}$ - $\mathrm{Pb}$ collisions at low $p_{T}$ and for the lowest quark mass, as expected due to the lower $x$ values probed into the nucleus for these cases. The dashed lines show the so called Shadowing-based calculations. It results from 

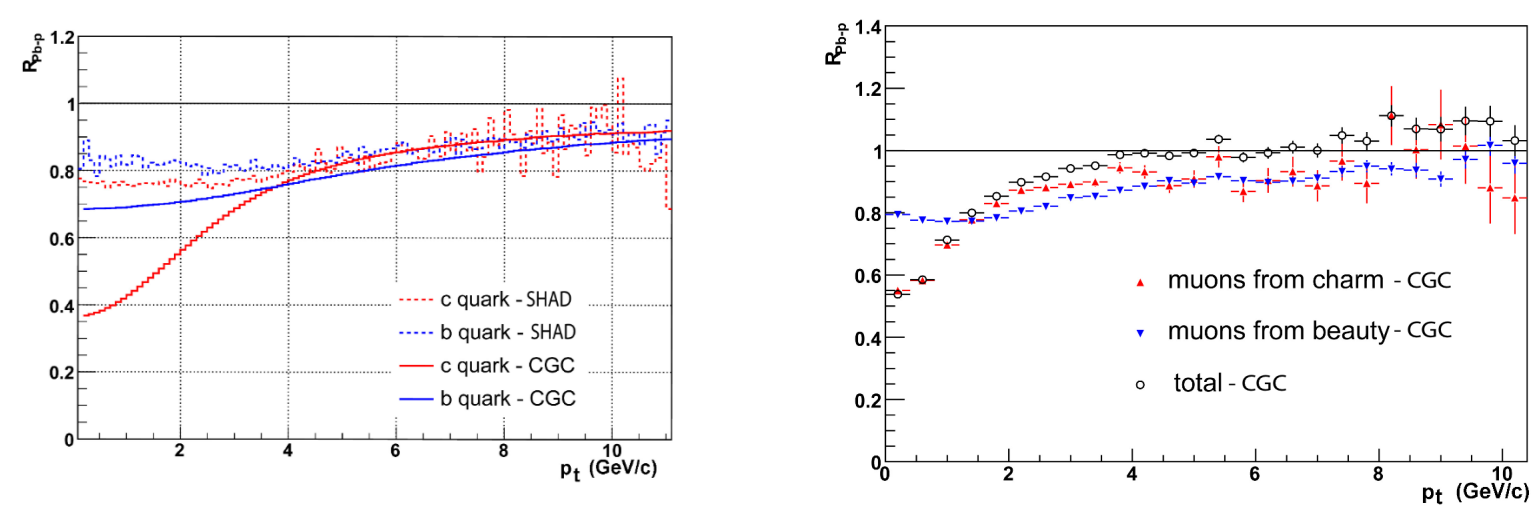

Figure 1: Nuclear modification factor as a function of $p_{T}$ for open heavy flavour (left plot) and single muon (right plot) production. See text for details.

a PYTHIA Monte Carlo generator where open heavy flavour production in p-p collisions were fitted to NLO pQCD calculations and shadowing effects were included by using the EKS98 nuclear parton distribution functions. The fragmentation of quarks into hadrons according to the Peterson function and finally the decay into muons of the produced mesons were carried out. The fragmentation process as well as the decay into muons soften the $p_{T}$ distribution. The right plot of Fig. 1 shows the resulted nuclear modification ratio for single muons. The Shadowing calculations, not displayed here, are approximately $0.8(0.9)$ for charm (beauty) at $p_{T} \leq 2 \mathrm{GeV} / \mathrm{c}$. A strongest suppression down to 0.55 in case of CGC calculations is observed for muons from open charm at $p_{T} \leq 2 \mathrm{GeV} / \mathrm{c}$. However the large uncertainties on the calculations at the $x$ value probed in the forward region may reduce the differences between the two models. The CGC calculations were implemented in a Monte Carlo generator and full simulations of the Forward Muon Spectrometer were carried out. It was shown that measuring saturation effects, visible at $p_{T} \leq 2 \mathrm{GeV} / \mathrm{c}$, is challenging: the muon trigger efficiency decreases below $2 \mathrm{GeV} / \mathrm{c}$ and single muon production is dominated by $\pi$ and $K$ coming from the interaction vertex at $p_{T} \leq 1.5 \mathrm{GeV} / \mathrm{c}$. This work is described in detail in [5].

\section{Forward $J / \psi$ production and detection performance with the ALICE Muon Spectrometer}

From the experimental point of view, it may be interesting now to consider the quarkonium production as a tool to search for saturation effects. The quarkonia can be indeed reconstructed in the Muon Spectrometer down to low $p_{T}$ with a good detector efficiency as shown, for the $J / \psi$ case, in the left plot of Fig. 2. Furthermore by fitting the invariant mass of the two detected opposite charge muons, one can easily estimate and subtract the background contamination to the $J / \psi$ yield [6]. As the saturation effect is larger for lower quark mass, only the $J / \psi$ production was considered. From Eq. 3.1, the $J / \psi$ production can be obtained according to the Color Evaporation Model (CEM) as the integration of the charm pair production over the pair invariant mass from the charm pair to the D meson pair production threshold. The right plot of Fig. 2 shows the calculated nuclear modification ratio for the CGC model (full line) as a function of $p_{T}$. A strong suppression down to 0.3 at low $p_{T}$ is seen in the forward region. The Shadowing calculation (dashed line) was 

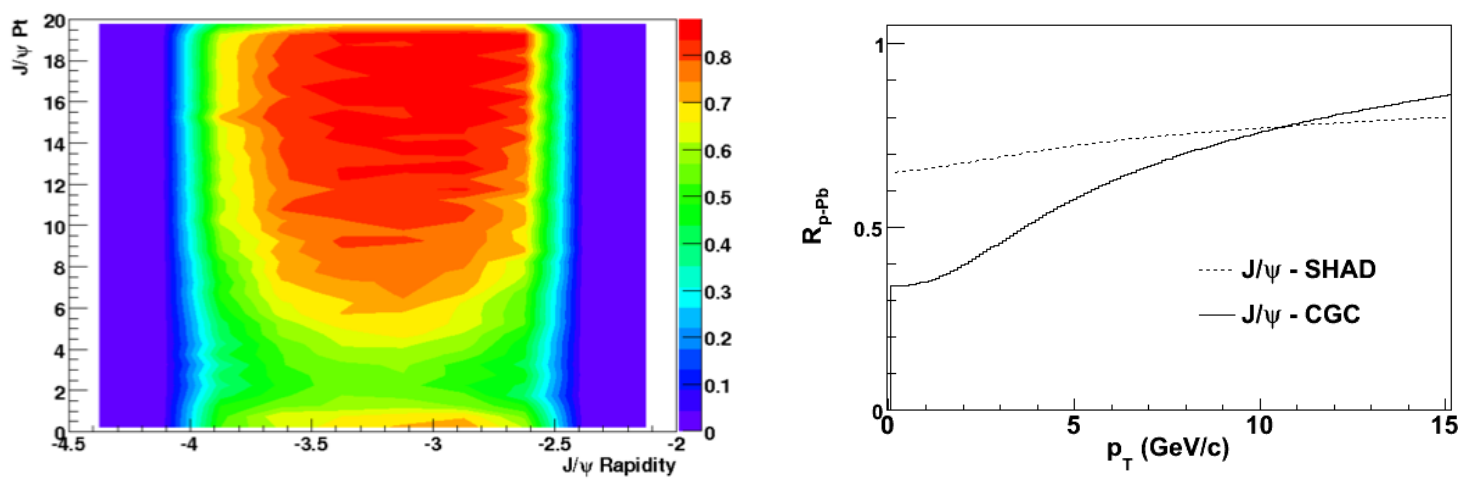

Figure 2: Left plot: efficiency for $J / \psi$ detection in the ALICE Muon Spectrometer. Right plot: nuclear modification factor as a function of $p_{T}$ for $J / \psi$ production. See text for details.

based on the CEM for the $y$ dependence and a fit on $J / \psi$ Tevatron data in p-p collisions for the $p_{T}$ dependence. In $\mathrm{p}-\mathrm{Pb}$ collisions, the shadowing factor from EKS98 was included. Although the CGC calculation shows a stronger suppression at $p_{T} \leq 10 \mathrm{GeV} / \mathrm{c}$, the uncertainties on the calculations may reduce the difference. A lower bound on the shadowing factor leads to a decrease of the nuclear modification factor by $30 \%$. Also CGC calculations present uncertainties according to the parameters chosen and are of the order of $30 \%$. The CGC calculations give us an estimate of the largest suppression expected in $\mathrm{p}-\mathrm{Pb}$ in comparison to $\mathrm{p}-\mathrm{p}$ collisions.

\section{Conclusion}

At small $x$, the gluon density in the nucleon is expected to saturate and the gluons to interact coherently with a probe. For this kinematic domain, the hadronic matter can be described within the Color Glass Condensate framework. In heavy ion collisions, measurements at RHIC already show some hints of gluon saturation effects but the LHC, with its much higher beam energy, should provide definitive tests of the CGC picture, in particular in $\mathrm{p}-\mathrm{Pb}$ collisions. Forward heavy flavour production is a suitable tool to search for saturation effects and measurements with the Muon Spectrometer in the ALICE experiment in $\mathrm{p}-\mathrm{Pb}$ collisions have been investigated. While single muon measurements seem to be quite challenging to quantify saturation effects, $J / \psi$ measurements appear to be more promising. Also $J / \psi$ production in ultra-peripheral $\mathrm{Pb}-\mathrm{Pb}$ collisions may be used as a tool to search for saturation effect. The forthcoming $\mathrm{Pb}-\mathrm{Pb}$ collisions expected for the end of 2010 and later on $\mathrm{p}-\mathrm{Pb}$ collisions at the LHC will shed light on the saturation regime.

\section{References}

[1] F. Gelis, E. Iancu, J. Jalilian-Marian and R. Venugopalan, arXiv:1002.0333, 2010

[2] BRAHMS Collaboration Phys.Rev.Lett. 93 (242303) 2004

[3] The ALICE Collaboration, K. Aamodt et al JINST 3 (S08002) 2008

[4] H. Fujii, F. Gelis, R. Venugopalan Nucl.Phys.A 780 (146-174) 2006

[5] A. Charpy et al., ALICE-INT-2009-043 EDMS id:1054935

[6] ALICE Collaboration et al, J.Phys.G: Nucl.Part.Phys. 32 (1295) 2006 